\title{
Explicit Time-Stepping Scheme for Radial Perfectly Matched Layers in Staggered Meshless Methods
}

\author{
Thomas Kaufmann and Christophe Fumeaux \\ The School of Electrical \& Electronic Engineering, \\ University of Adelaide, Adelaide, SA, 5005, Australia. \\ Email: thomas.kaufmann@adelaide.edu.au
}

\begin{abstract}
Meshless methods have attracted attention recently as versatile algorithms in computational electromagnetics. The Radial Point Interpolation Method (RPIM) is one particular type of meshless method based on radial basis functions implemented in a collocation time-domain framework. The possibility of free node placement allows conformal modeling of complex geometries with potentially significantly lower memory consumption and higher accuracy than the finite-difference time-domain (FDTD) method. Open boundaries have been implemented in the form of uniaxial perfectly matched layers (PMLs). A natural next step for this method is the implementation of locally conformal PMLs. A special case of conformal PMLs are the radial PMLs, which are characterized by an anisotropy in radial direction. In this paper, a methodology to obtain explicit update equations for the electric and magnetic field components from an originally implicit dependence of the magnetic field is presented.
\end{abstract}

\section{INTRODUCTION}

In computational electromagnetics (EM), meshless methods represent a relatively new class of techniques for solving the Maxwell's equations. Introduced earlier in other fields of physics [1], this type of methods show some striking properties that open new perspectives for many advanced applications in EM. Instead of solving a problem on an unstructured mesh topology or an orthogonal grid, the field values are stored at arbitrarily placed node locations. No a priori connectivity between the nodes exists, but sophisticated basis functions form the relationship between the nodes. This concept provides flexibility in the discretization for conformal and multi-scale modeling. Furthermore, it facilitates adaptation of node distributions for optimization or for the modeling of fast dynamic processes. This makes the class of meshless methods highly attractive for the simulation of nano-optics and multi-physics problems.

Many very different formulations of meshless methods exist, some closer to finite-difference methods [2], others closer to finite element or boundary element methods [3]. In this paper, we focus on a differential collocation method based on radial basis functions (RBFs). A special form of RBF methods is the radial point interpolation method (RPIM) [4], which yields an explicit scheme to solve Maxwell's equations in the presence of isotropic materials [5]. The method was introduced for a two-dimensional transverse electric case in [6] and was extended to a 3D formulation in [7]. After the observation of longtime stability issues in an eigenvalue formulation [8], an unconditionally stable formulation was introduced in [9].
Alternatively, a formulation that uses Laguerre polynomials to model the time variable was presented in [10].

An important feature for domain-discretization methods is the capability to solve open problems, e.g. radiation or scattering problems. This type of applications require efficient absorbing boundary conditions to truncate free-space in a finite computational domain. In computational EM, perfectly matched layers (PMLs) have become the standard for efficient domain truncation [11]. PMLs have recently been introduced to the RPIM scheme in a uniaxial formulation [12].

A natural step is the extension to radial PMLs [13], [14]. In this paper, a series of mathematical manipulations to achieve an explicit time stepping scheme for radially anisotropic PMLs are introduced. The scheme is validated through numerical experiments.

\section{RAdial Point Interpolation Method}

The radial basis point interpolation method was first introduced in computational mechanics in [4] and employs a combination of radial and polynomial basis functions. The field component $u$ at position $\mathbf{x}=(x, y)$ is approximated as follows:

$$
u(\mathbf{x}) \approx \sum_{n=1}^{N} r_{n}(\mathbf{x}) a_{n}+\sum_{m=1}^{M} p_{m}(\mathbf{x}) b_{m}
$$

with $r_{n}(\mathbf{x})$ the radial basis function and $p_{m}(\mathbf{x})=\left[\begin{array}{lll}1 & x & y\end{array}\right]$ a first-order polynomial basis function. $N$ is the number of points inside a local support domain (Fig. 1) which represents the extent of the basis functions. Often, the support domain encloses 8-12 neighbors in a $2 \mathrm{D}$ setting. The interpolation coefficients $a_{n}, b_{n}$ are calculated in a preprocessing state in a point-matching procedure [5]. Eventually, a set of shape functions is computed through an efficient local matrix inversion and is expressed as

$$
u(\mathbf{x}) \approx \Psi(\mathbf{x})\left(\begin{array}{c}
u_{1} \\
\vdots \\
u_{N}
\end{array}\right),
$$

where $u_{i}$ are the field component values at the surrounding node locations in the support domain. Also, spatial derivatives 


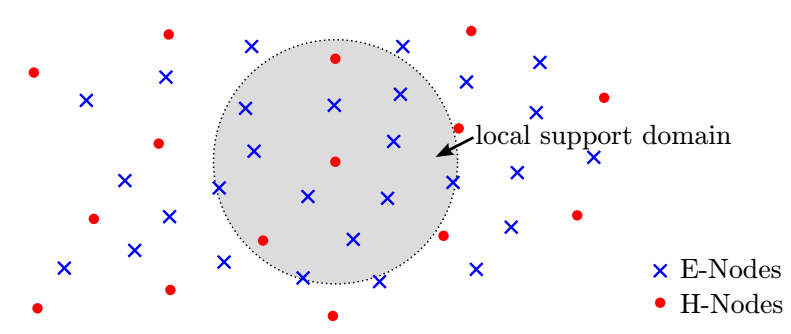

Fig. 1. Staggered node distribution of electric and magnetic nodes. A local support domain limits the extend of the basis functions.

in $\kappa=x, y$ direction can be approximated through

$$
\partial_{\kappa} u(\mathbf{x}) \approx \partial_{\kappa} \Psi(\mathbf{x})\left(\begin{array}{c}
u_{1} \\
\vdots \\
u_{N}
\end{array}\right) .
$$

Gaussian radial basis functions centered at node location $\mathbf{x}_{n}=$ $\left(x_{n}, y_{n}\right)$

$$
r_{n}(\mathbf{x})=\exp \left(-\alpha_{c}\left(\frac{\left|\mathbf{x}_{n}-\mathbf{x}\right|}{d_{c}}\right)^{2}\right)
$$

with the average node distance $d_{c}$ as a normalization term and a global shape parameter $\alpha_{c}$ prove to be a good choice in terms of accuracy and speed. The shape parameter $\alpha_{c}$ determines the width of the RBFs. It tunes the condition number for the local matrix inversion and influences the accuracy of the local interpolation [8].

Similar to the Yee grid in the finite-difference time-domain (FDTD) method, a staggered node distribution based on a Voronoi tessellation [15] is employed to obtain two separate sets of electric and magnetic field nodes (E-, H-Nodes). Fig. 1 illustrates such a node distribution. In this case, two separate sets of shape functions for the E- and H-Nodes are computed in the preprocessing.

The time-domain implementation of the meshless RPIM scheme for Maxwell's equations has been summarized in [5]. The solution in the time domain is found through a leap-frog time iteration. In this paper, a formulation of the transverseelectric (TE) mode in two dimensions is implemented for demonstration, but it can be generalized to $3 \mathrm{D}$.

\section{RADIAL PML}

In PML implementations, layers of an artificial lossy material engineered to be matched to the surrounding space absorb outgoing EM energy on the boundary of the computational domain. Due to the orthogonal grid arrangement in the standard FDTD algorithm, uniaxial PMLs are usually implemented in computational domains shaped as a rectangular box. In the time-domain RPIM scheme, uniaxial PMLs have been introduced in [5], [12].

The use of PMLs in rectangular boxes does not exploit the capabilities of conformal modeling offered by meshless methods. For many problems, it could be more efficient to use a spherical (or cylindrical in 2D) domain with PMLs cladded on its inner surface. A radial PML arrangement might result in reduction of the computational volume and in a better absorption performance, as incidence will be typically occurring closer to normal. For the finite-volume time-domain (FVTD) method, such a formulation has been introduced in [13], [14]. An implementation for the ADI FDTD method has been presented in [16], where the implicit nature of the formulation could be directly taken into account in the ADI scheme.

The locally radial type of PML presented in the following is based on a uniaxial Maxwellian absorber [17] with a local coordinate transformation applied to allow for absorption in arbitrary directions. For TE modes, the governing equations take the following form [13]:

$$
\begin{aligned}
\partial_{t} K_{r}= & \frac{1}{\mu}\left[-\zeta_{r} \sin \varphi \partial_{x} E_{z}+\zeta_{r} \cos \varphi \partial_{y} E_{z}\right] \\
\partial_{t} H_{x}= & -\frac{1}{\mu} \partial_{y} E_{z}+\zeta_{r} H_{y} \cos \varphi \sin \varphi-\zeta_{r} H_{x} \sin ^{2} \varphi \\
& -K_{r} \cos \varphi \\
\partial_{t} H_{y}= & \frac{1}{\mu} \partial_{x} E_{z}+\zeta_{r} H_{x} \cos \varphi \sin \varphi-\zeta_{r} H_{y} \cos ^{2} \varphi-K_{r} \sin \varphi \\
\partial_{t} E_{z}= & \frac{1}{\varepsilon}\left[\partial_{x} H_{y}-\partial_{y} H_{x}\right]-\zeta_{r} E_{z} .
\end{aligned}
$$

The radial magnetic polarization current $K_{r}$ is solved in the auxiliary differential equation (5a). The absorption coefficient $\zeta_{r}$ is tapered radially to minimize numerical discretization errors. The angular position $\varphi$ of the absorber axis is measured from the $x$-axis and its dependence results from the local coordinate transformation.

Due to anisotropic material properties of the PML, the time derivatives of the magnetic field in (5b) and (5c) depend on the other magnetic field components. When written in vector form, the magnetic field can be expressed as

$$
\partial_{t}\left(\begin{array}{l}
H_{x} \\
H_{z}
\end{array}\right)=\mathbf{A}\left(\begin{array}{l}
H_{x} \\
H_{z}
\end{array}\right)+\mathbf{B} K_{r}+\mathbf{C} E_{z}
$$

with the parameter matrix $\mathbf{A}$ and vector $\mathbf{B}$

$$
\begin{aligned}
& \mathbf{A}=\left(\begin{array}{cc}
-\zeta_{r} \sin ^{2} \varphi & \zeta_{r} \cos \varphi \sin \varphi \\
\zeta_{r} \cos \varphi \sin \varphi & -\zeta_{r} \cos ^{2} \varphi
\end{array}\right) \\
& \mathbf{B}=\left(\begin{array}{l}
-\cos \varphi \\
-\sin \varphi
\end{array}\right)
\end{aligned}
$$

and an operator vector

$$
\mathbf{C}=\left(\begin{array}{c}
-\frac{1}{\mu} \partial_{y} \\
\frac{1}{\mu} \partial_{x}
\end{array}\right) .
$$

In the predictor-corrector time discretization scheme employed in the FVTD method [13], the electric and magnetic field nodes are co-located in time, and that naturally yields an explicit time stepping.

When the field components are located in a time-staggered arrangement though, (5b) and (5c) become implicit. This has 


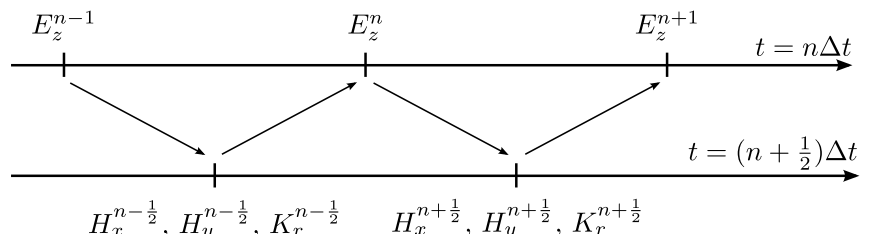

Fig. 2. Illustration of the discrete staggered time variable.

not been an issue in the past since the standard explicit FDTD method did not employ non-orthogonal PMLs. For the timedomain RPIM algorithm, a new methodology is required to obtain efficient explicit update equations.

\section{EXPLICIT UPDATE EQUATIONS}

In the following, the derivation of new update equations for the 2D TE-mode with anisotropic radial PMLs is shown. First the time variable is discretized and brought into explicit form. Then the space variables are discretized at all node locations using the RPIM scheme. A focus is laid on the magnetic field expressions, as they are the most affected by the mathematical manipulations towards the explicit TE formulation.

The time variable is discretized in staggered regular time steps as illustrated in Fig. 2 and solved through a leap-frog time integration. The temporal derivatives are approximated using central differences

$$
\frac{u^{n+\frac{1}{2}}-u^{n-\frac{1}{2}}}{\Delta t}=f\left(u^{n}\right) .
$$

When applied to (6), this results in the following semi-discrete equation

$$
\begin{array}{r}
\left(\begin{array}{c}
H_{x} \\
H_{z}
\end{array}\right)^{n+\frac{1}{2}}-\left(\begin{array}{c}
H_{x} \\
H_{z}
\end{array}\right)^{n-\frac{1}{2}}=\frac{\Delta t}{2} \mathbf{A} \\
\left(\left(\begin{array}{c}
H_{x} \\
H_{z}
\end{array}\right)^{n+\frac{1}{2}}+\left(\begin{array}{c}
H_{x} \\
H_{z}
\end{array}\right)^{n-\frac{1}{2}}\right) \\
+\Delta t \mathbf{B} K_{r}^{n}+\Delta t \mathbf{C} E_{z}^{n} .
\end{array}
$$

The right-hand side is evaluated at time step $n$, therefore a time averaging of the future and past field magnetic values is performed. The field values at time step $n+\frac{1}{2}$ are brought to the left side of the equation:

$$
\begin{aligned}
\left(\mathbf{I}-\frac{\Delta t}{2} \mathbf{A}\right)\left(\begin{array}{c}
H_{x} \\
H_{z}
\end{array}\right)^{n+\frac{1}{2}}= & \left(\mathbf{I}+\frac{\Delta t}{2} \mathbf{A}\right)\left(\begin{array}{c}
H_{x} \\
H_{z}
\end{array}\right)^{n-\frac{1}{2}} \\
& +\Delta t \mathbf{B} K_{r}^{n}+\Delta t \mathbf{C} E_{z}^{n}
\end{aligned}
$$

In a next step to obtain explicit update equations, the matrix $\left(\mathbf{I}-\frac{\Delta t}{2} \mathbf{A}\right)$ is eliminated from the left side by a multiplication of its inverse with the whole equation

$$
\begin{aligned}
& \left(\begin{array}{c}
H_{x} \\
H_{z}
\end{array}\right)^{n+\frac{1}{2}}=\left(\mathbf{I}-\frac{\Delta t}{2} \mathbf{A}\right)^{-1}\left(\mathbf{I}+\frac{\Delta t}{2} \mathbf{A}\right)\left(\begin{array}{c}
H_{x} \\
H_{z}
\end{array}\right)^{n-\frac{1}{2}} \\
& +\Delta t\left(\mathbf{I}-\frac{\Delta t}{2} \mathbf{A}\right)^{-1} \mathbf{B} K_{r}^{n}+\Delta t\left(\mathbf{I}-\frac{\Delta t}{2} \mathbf{A}\right)^{-1} \mathbf{C} E_{z}^{n}
\end{aligned}
$$

which can be summarized as

$$
\left(\begin{array}{c}
H_{x} \\
H_{z}
\end{array}\right)^{n+\frac{1}{2}}=\mathbf{D}\left(\begin{array}{c}
H_{x} \\
H_{z}
\end{array}\right)^{n-\frac{1}{2}}+\mathbf{E} K_{r}^{n}+\mathbf{F} E_{z}^{n} .
$$

The elements of the matrices in (14) are calculated through some simple analytical operations on $2 \times 2$ matrices and the final expressions are given in the following. The symmetric parameter matrix $\mathbf{D}=\left[d_{i j}\right]_{2 \times 2}$ is a full matrix

$$
\begin{aligned}
& d_{11}=\frac{2+\zeta_{r} \Delta t\left(-\sin ^{2} \varphi+\cos ^{2} \varphi\right)}{2+\zeta_{r} \Delta t} \\
& d_{12}=\frac{2 \zeta_{r} \Delta t \sin \varphi \cos \varphi}{2+\zeta_{r} \Delta t} \\
& d_{21}=d_{12} \\
& d_{22}=\frac{2+\zeta_{r} \Delta t\left(\sin ^{2} \varphi-\cos ^{2} \varphi\right)}{2+\zeta_{r} \Delta t}
\end{aligned}
$$

The parameter vector $\mathbf{E}=\left[e_{i}\right]_{2 \times 1}$ is expressed as

$$
\begin{aligned}
& e_{1}=-\Delta t \cos \varphi \\
& e_{2}=-\Delta t \sin \varphi .
\end{aligned}
$$

This surprisingly simple expression can explained by the fact that the parameter vector $\mathbf{B}$ is an eigenvector of $\left(\mathbf{I}-\frac{\Delta t}{2} \mathbf{A}\right)$ with eigenvalue $\lambda=1$.

The modified operator vector $\mathbf{F}=\left[f_{i}\right]_{2 \times 1}$ consist of spatial derivatives in the $x, y$-direction. In contrast to the original operator vector $\mathbf{C}$ from (9), $\mathbf{F}$ contains derivatives in both coordinate axis in each element. The operator vector is reformulated with the spatial derivatives separately weighted with a parameter matrix $\mathbf{G}=\left[g_{i j}\right]_{2 \times 2}$ such that

$$
\mathbf{F}=\mathbf{G}\left(\begin{array}{l}
\partial_{x} \\
\partial_{y}
\end{array}\right)
$$

Then, $\mathbf{F}$ becomes

$$
\begin{aligned}
f_{1} & =\frac{\Delta t}{\mu} \frac{\zeta_{r} \Delta t \sin \varphi \cos \varphi \partial_{x}-\left(2+\zeta_{r} \Delta t \cos ^{2} \varphi\right) \partial_{y}}{2+\zeta_{r} \Delta t} \\
& =\frac{\Delta t}{\mu}\left[g_{11} \partial_{x}+g_{12} \partial_{y}\right] \\
f_{2} & =\frac{\Delta t}{\mu} \frac{\left(2+\zeta_{r} \Delta t \sin ^{2} \varphi\right) \partial_{x}-\zeta_{r} \Delta t \sin \varphi \cos \varphi \partial_{y}}{2+\zeta_{r} \Delta t} \\
& =\frac{\Delta t}{\mu}\left[g_{21} \partial_{x}+g_{22} \partial_{y}\right] .
\end{aligned}
$$

The explicit expressions for the electric field are obtained straightforwardly in the TE formulation, considering that as for the magnetic field, a time-averaging for the loss term in the transverse electric field $E_{z}$ is performed as described in [18]. For an extension of the formulation to spherical or cylindrical PMLs in a three dimensional setting however, mathematical transformations as shown here for the magnetic fields would be required for all fields and would result in similar expressions.

Considering now the problem including the discretization in space, the field values are evaluated at the two sets of E- and $\mathrm{H}$-nodes. The spatial derivatives in the operator matrix (18) 
are approximated with the RPIM method (3). The parameter matrices $\mathbf{D}$ and $\mathbf{G}$ as well as the vector $\mathbf{E}$ only depend on the node location and can be evaluated for each node separately. The following explicit update equations for the node $i$ result

$$
\begin{aligned}
& K_{r, i}^{n+\frac{1}{2}}=K_{r, i}^{n-\frac{1}{2}}-\frac{\Delta t \zeta_{r, i}}{\mu_{i}} . \\
& {\left[-\sin \varphi_{i} \sum_{j=1}^{n_{A_{s}}} \partial_{y} \Phi_{E, i}^{j} E_{z, j}^{n}+\cos \varphi_{i} \sum_{j=1}^{n_{A_{s}}} \partial_{x} \Phi_{E, i}^{j} E_{z, j}^{n}\right]} \\
& H_{x, i}^{n+\frac{1}{2}}=d_{11, i} H_{x, i}^{n-\frac{1}{2}}+d_{12, i} H_{y, i}^{n-\frac{1}{2}}+e_{11, i} K_{r, i}^{n}+ \\
& \frac{\Delta t}{\mu_{i}}\left[g_{11, i} \sum_{j=1}^{n_{A_{s}}} \partial_{x} \Phi_{E, i}^{j} E_{z, j}^{n}+g_{12, i} \sum_{j=1}^{n_{A_{s}}} \partial_{y} \Phi_{E, i}^{j} E_{z, j}^{n}\right] \\
& H_{y, i}^{n+\frac{1}{2}}=d_{21, i} H_{x, i}^{n-\frac{1}{2}}+d_{22, i} H_{y, i}^{n-\frac{1}{2}}+e_{22, i} K_{r, i}^{n}+ \\
& \frac{\Delta t}{\mu_{i}}\left[g_{21, i} \sum_{j=1}^{n_{A_{s}}} \partial_{x} \Phi_{E, i}^{j} E_{z, j}^{n}+g_{22, i} \sum_{j=1}^{n_{A_{s}}} \partial_{y} \Phi_{E, i}^{j} E_{z, j}^{n}\right] \\
& E_{z, i}^{n+1}=\frac{2-\zeta_{r, i} \Delta t}{2+\zeta_{x} \Delta t} E_{z, i}^{n}+\frac{2 \Delta t}{\varepsilon_{i}\left(2+\zeta_{r, i} \Delta t\right)} . \\
& {\left[\sum_{j=1}^{n_{A_{s}}} \partial_{x} \Phi_{H, i}^{j} H_{y, j}^{n+\frac{1}{2}}-\sum_{j=1}^{n_{A_{s}}} \partial_{y} \Phi_{H, i}^{j} H_{x, j}^{n+\frac{1}{2}}\right] .}
\end{aligned}
$$

The values of the magnetic polarization currents at time steps $n$ in (19b) and (19c) are again obtained through time-averaging

$$
K_{r, i}^{n}=\frac{K_{r, i}^{n-\frac{1}{2}}+K_{r, i}^{n+\frac{1}{2}}}{2} .
$$

To minimize the discretization errors a radially dependent polynomial profile of order $m$ is applied to the absorption term $\zeta_{r}$ at radial location $\rho$

$$
\zeta_{r}(\rho)=\zeta_{0}\left(\frac{\rho-\rho_{0}}{\delta}\right)^{m}
$$

This expression applies for a PML with a thickness $\delta$, starting at radius $\rho_{0} . \zeta_{0}$ is the maximum absorption coefficient. Quantitatively, this value can be chosen so that the theoretically expected reflection at normal incidence [11]

$$
\Gamma_{t h}(0)=\exp \left(-\frac{2 \zeta \delta}{(m+1) c}\right) .
$$

This is set here at $\Gamma_{t h}=-80 \mathrm{~dB}$ with the polynomial profile selected as $m=2$.

The correctness of these derived formulas can be partly tested by checking that the original update equations are restored when setting the absorption term $\zeta_{r}=0$. In that case, the parameter matrix $\mathbf{D}$ becomes the identity matrix, the magnetic polarization current remains zero over all time steps, and the parameter matrix

$$
\mathbf{G}=\left(\begin{array}{cc}
0 & -1 \\
1 & 0
\end{array}\right)
$$

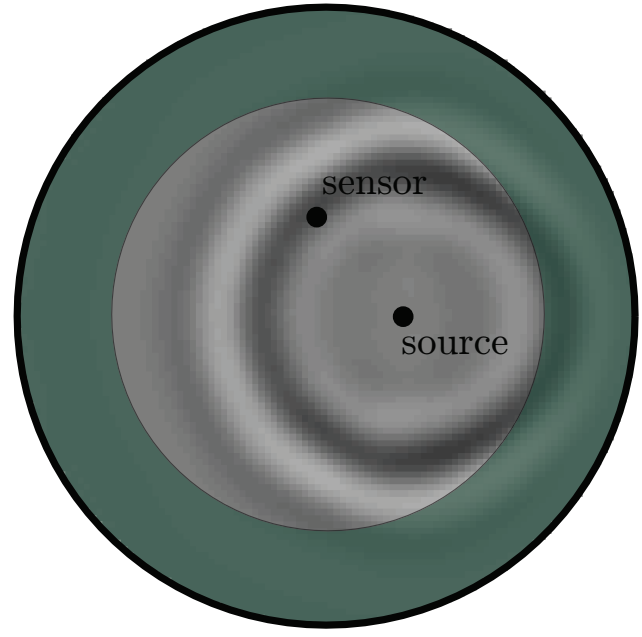

Fig. 3. Electric field distribution after 123 time steps with energy injected in a line current. The black dots mark the position of the line current and the sensor node.

corresponds to the discretized curl operation when multiplied with the operator vector $\left(\begin{array}{ll}\partial_{x} & \partial_{y}\end{array}\right)^{T}$. All update equations are then independent of $\varphi$. Also, the electric field update equation (19d) reverts to the standard expression. Hence, in free space, the standard update equations are successfully reconstructed from (19).

The explicit update equations do not require a matrix inversion at every time step, but rather contain a number of geometrical operations. All the required geometrical terms can be calculated in a preprocessing stage. For best efficiency, the application of these RPIM PML equations can be limited to the absorbing layers, whereas the standard RPIM is kept in the rest of the computational domain. The steps introduced in this article can easily be extended to other types of anisotropic material properties with arbitrary axis orientation. Also it should be noted that the methodology introduced here is not limited to the RPIM shape functions. Other meshless interpolation methods could be used alternatively in a staggered time stepping.

\section{NUMERICAL EXPERIMENT}

The radial PML is demonstrated in a numerical example. For a cylindrical domain, a line current at an off-center location radiates energy with a broad frequency bandwidth. A radial PML absorbs the energy at oblique angles of incidence. The discretization is chosen such that the average node distance corresponds to $\lambda_{\max } / 10$ and $\lambda_{\min } / 20$ at the largest and smallest simulated wavelengths, respectively. The width of the PML material is set to $0.75 \lambda_{\max }$. To calculate the reflected electric field, a reference solution is computed in a larger domain.

Fig. 3 shows the radiated pulse after 123 time steps, with the circular wave front partly being absorbed by the PML. The normalized total and reflected electric fields at the sensor location are shown in Fig. 4. The magnitude of the reflected field at the sensor location is around $-60 \mathrm{~dB}$. This is an 


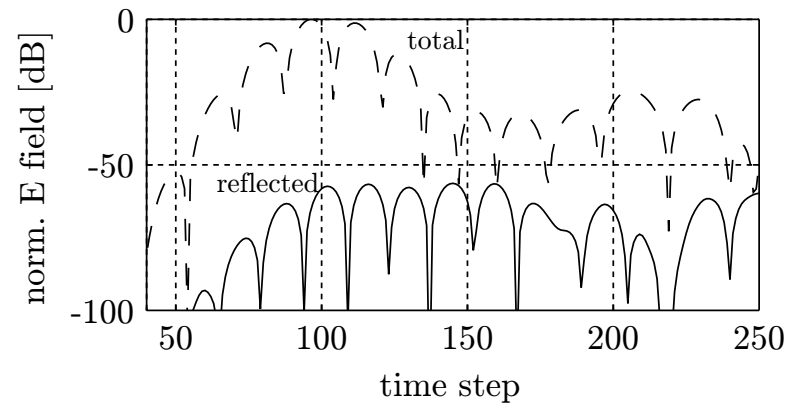

Fig. 4. Normalized total and reflected electric field at the sensor location.

expected value considering that most of the power impinges on the PML at angles well off normal incidence. The computation time for this problem on a standard PC was $1.44 \mathrm{~s}$ - using the free-space update equations, the simulation was $1.14 \mathrm{~s}$.

\section{CONCLUSION}

A methodology for meshless methods that employ a staggered time stepping scheme is introduced in the framework of radial PMLs while keeping explicit update equations. This greatly increases the simulation speed compared to the requirement of an implicit scheme, where a matrix has to be inverted at each time step. Also, the radial PMLs limit the requirement on the computational domain size for problems with spherical wave solutions, e.g. radiating problems, as this absorbing boundary can be placed closer to the objects. The effectiveness of the radial PMLs has been confirmed in numerical experiments.

The steps introduced can be extended to other types of radially anisotropic materials and to other staggered meshless methods. A natural next step is the extension towards spherical PMLs in a three-dimensional setting.

\section{REFERENCES}

[1] V. P. Nguyen, T. Rabczuk, S. Bordas, and M. Duflot, "Meshless methods: A review and computer implementation aspects," Mathematics and Computers in Simulation, vol. 79, no. 3, pp. 763-813, 2008.

[2] E. Kansa, "Multiquadrics - A scattered data approximation scheme with applications to computational fluid-dynamics - I surface approximations and partial derivative estimates," Computers \& Mathematics with Applications, vol. 19, no. 8-9, pp. 127-145, 1990
[3] T. Belytschko, D. Organ, and Y. Krongauz, "A coupled finite elementelement-free Galerkin method," Computational Mechanics, vol. 17, pp. 186-195, 1995.

[4] G. R. Liu and Y. T. Gu, An Introduction to Meshfree Methods and their Programming. Dortrecht, The Netherlands: Springer, 2005.

[5] T. Kaufmann, Y. Yu, C. Engström, Z. Chen, and C. Fumeaux, "Recent developments of the meshless radial point interpolation method for timedomain electromagnetics," International Journal of Numerical Modelling: Electronic Networks, Devices and Fields, 2012, to be published.

[6] T. Kaufmann, C. Fumeaux, and R. Vahldieck, "The meshless radial point interpolation method for time-domain electromagnetics," in IEEE MTT-S International Microwave Symposium Digest. Atlanta, GA, USA: IEEE, Jun. 2008, pp. 61-65.

[7] Y. Yu and Z. D. Chen, "A 3-D radial point interpolation method for meshless time-domain modeling," IEEE Trans. Microwave Theory and Techn., vol. 57, no. 8, pp. 2015-2020, Aug. 2009.

[8] T. Kaufmann, C. Engström, C. Fumeaux, and R. Vahldieck, "Eigenvalue analysis and longtime stability of resonant structures for the meshless radial point interpolation method in time domain," IEEE Trans. Microwave Theory and Techn., vol. 58, no. 12, pp. 3399-3408, December 2010.

[9] Y. Yu and Z. D. Chen, "Towards the development of an unconditionally stable time-domain meshless method," IEEE Trans. Microwave Theory and Techn., vol. 58, no. 3, pp. 578-586, Mar. 2010.

[10] X. Chen, Z. Chen, Y. Yu, and D. Su, "An unconditionally stable radial point interpolation meshless method with Laguerre polynomials," IEEE Trans. Antennas and Propagation, vol. 59, no. 10, pp. 3756-3763, Oct. 2011.

[11] J.-P. Bérenger, "Perfectly matched layer (PML) for computational electromagnetics," Synthesis Lectures on Computational Electromagnetics, vol. 2, no. 1, pp. 1-117, 2007.

[12] Y. Yu and Z. D. Chen, "Meshless RPIM modeling of open-structures using PMLs," in IEEE MTT-S International Microwave Symposium Digest, May 2010, pp. 97-100.

[13] K. Sankaran, C. Fumeaux, and R. Vahldieck, "Uniaxial and radial anisotropy models for finite-volume Maxwellian absorber," IEEE Trans. Microwave Theory and Techn., vol. 54, no. 12, pp. 4297-4304, Dec. 2006.

[14] C. Fumeaux, K. Sankaran, and R. Vahldieck, "Spherical perfectly matched absorber for finite-volume 3-D domain truncation," IEEE Trans. Microwave Theory and Techn., vol. 55, no. 12, pp. 2773-2781, 2007.

[15] C. B. Barber, D. P. Dobkin, and H. Huhdanpaa, "The Quickhull algorithm for convex hulls," ACM Trans. Math. Softw., vol. 22, no. 4, pp. 469-483, 1996.

[16] H.-X. Zheng and K. W. Leung, "Radial perfectly matched layer for the ADI-FDTD method," Microwave and Wireless Components Letters, IEEE, vol. 19, no. 7, pp. $425-427$, july 2009.

[17] R. Ziolkowski, "Time-derivative Lorentz material model-based absorbing boundary condition," IEEE Trans. Microwave Theory and Techn., vol. 45 , no. 10 , pp. 1530-1535, 2002.

[18] S. Gedney, "An anisotropic perfectly matched layer-absorbing medium for the truncation of FDTD lattices," IEEE Trans. Microwave Theory and Techn., vol. 44, no. 12, pp. 1630-1639, Dec. 1996. 